\title{
Analysis of active components in Salvia miltiorrhiza injection based on vascular endothelial cell protection
}

\author{
JIE SHEN ${ }^{1}$ \\ KAI YANG ${ }^{2}$ \\ CAIHUA SUN ${ }^{1}$ \\ MINXIA ZHENG ${ }^{1 *}$ \\ ${ }^{1}$ The First Affiliated Hospital \\ of Zhejiang Chinese \\ Medical University, Hangzhou \\ Zhejiang Province - 310 006, China \\ ${ }^{2}$ The First Affiliated Hospital \\ of Medical School of Zhejiang \\ University, Hangzhou \\ Zhejiang Province - 310 003, China
}

Accepted May 14, 2014

\begin{abstract}
Correlation analysis based on chromatograms and pharmacological activities is essential for understanding the effective components in complex herbal medicines. In this report, HPLC and measurement of antioxidant properties were used to describe the active ingredients of Salvia miltiorrhiza injection (SMI). HPLC results showed that tanshinol, protocatechuic aldehyde, rosmarinic acid, salvianolic acid B, protocatechuic acid and their metabolites in rat serum may contribute to the efficacy of SMI. Assessment of antioxidant properties indicated that differences in the composition of serum powder of SMI caused differences in vascular endothelial cell protection. When bivariate correlation was carried out it was found that salvianolic acid B, tanshinol and protocatechuic aldehyde were active components of SMI because they were correlated to antioxidant properties.
\end{abstract}

Keywords: Salvia miltiorrhiza injection, HPLC, antioxidant activity, correlation analysis, salvianolic acid

Herbal medicines and their derivatives are widely used as therapeutics in many countries (1). Radix Salviae miltiorrhizae, called "Danshen" in China, is one of the most important traditional Chinese herbs and is accepted as a health product in western countries owing to its remarkable and reliable biological activities, especially in the treatment of cardiovascular disorders (2). Salvia miltiorrhiza products commonly include tablets, pills, and injections (3). Formulation of Salvia miltiorrhiza dripping pills has been certified by the US FDA Phase II clinical trials. Salvia miltiorrhiza injection (SMI) is believed to be another potential herbal product and has been the subject of recent studies.

SMI made from the aqueous extract of Radix Salviae miltiorrhizae is widely prescribed to patients with coronary disease, unstable angina, brain hemorrhage, cerebral thrombosis and cerebrovascular diseases in Chinese clinics (3-5). In addition, it is also applied to treat other diseases, such as liver dysfunction, renal deficiency and diabetic vascular complications (4). Studies of SMI showed that phenolic acids are beneficial compounds, responsible for therapeutic efficacy (5-8). Moreover, it is worth noting that phenolic acids of Radix Salviae miltiorrhizae such as salvianolic acid A, salvianolic acid B and rosmarinic acid have been reported to exhibit antioxidant properties and were protective against peroxidative

\footnotetext{
*Correspondence; e-mail address: slightsusu@163.com
} 
damage $(9,10)$. Other studies showed that the water soluble extract of Radix Salviae miltiorrhizae exhibited high reactive oxygen species scavenging activity $(11,12)$. Many methods have been developed for chemical and pharmacological investigations of SMI, including chemical analysis with HPLC-UV, HPLC-MS, UPLC-MS, HPLC-CL and in vivo / in vitro pharmacological studies (13-18). However, there are few reports on ingredient analysis of SMI correlated to its biological activity.

Though we have already reported a simultaneous detection of seven phenolic acids in SMI (13), investigations in which phenolic acids have higher correlation to cardiovascular protection are more meaningful but have not been published yet. Therefore, in this paper, we present a study correlating phenolic acids in serum powder of SMI to its vascular endothelial cell protection by the use of bivariate correlation analysis (e.g., ref. 19).

\section{EXPERIMENTAL}

\section{Chemicals}

Standards including tanshinol (99.8\%), protocatechuic aldehyde $(99.8 \%)$, protocatechuic acid $(99.8 \%)$, rosmarinic acid $(99.8 \%)$ and salvianolic acid B $(99.5 \%)$ were purchased from the National Institute for the Control of Pharmaceutical and Biological Products (China). Salvianolic acid A was isolated from the aqueous extract of Radix Salviae miltiorrhizae and was identified on the basis of mass spectrum data; its purity was over $97 \%$ (by HPLC analysis). SMI was kindly supplied by Qinchunbao Pharmaceutical Co., Ltd. (China). Methanol, acetonitrile and formic acid (Merck, Germany) were of HPLC grade. Cholestan-3 $\beta, 5 \alpha, 6 \beta$-triol (triol), superoxide dismutase (SOD) kits and 1,1-diphenyl-2-picrylhydrazyl (DPPH) were purchased from Sangon Biotech Co., Ltd. (China). Materials used for cell experiments including human umbilical vein endothelial cells (HUVECs), Dulbecco's modified Eagle's medium (DMEM), fetal calf serum (FCS), recombinant human basic fibroblast growth factor (bFGF), bovine serum albumin (BSA) and 4',6-diamidino-2-phenylindole (DAPI) were also purchased from Sangon Biotech Co., Ltd. Deionized water used throughout the experiments was produced using a Mill-Q water purification system (Millipore, USA).

\section{Animals and blood samples}

Female Sprague Dawley rats (clean grade), weighting 220-250 g, were obtained from the Zhejiang Academy of Medical Sciences (Hangzhou, China). The studies were approved by the Animal Ethics Committee of Zhejiang Chinese Medical University (Hangzhou, China). Rats were fasted overnight, with free access to water, before the experiment. They were then randomly assigned into six groups (groups $1-6, n=6$ each). Groups $1-6$ were given SMI, $630 \mathrm{mg} \mathrm{kg}^{-1}$ by intravenous injection.

Blood samples $(0.2 \mathrm{~mL})$ were collected from the ophthalmic venous plexus in heparinized polythene tubes just before drug injection and 5 (group 1), 10 (group 2), 15 (group 3), 20 (group 4), 25 (group 5) and 30 (group 6) min after injection. Serum was separated quickly using centrifugation at $1600 \times g$ for $10 \mathrm{~min}$. One $\mathrm{mL}$ acetone was added to serum and centrifuged at $3000 \times g$ for $10 \mathrm{~min}$ to remove proteins. The supernatant was concentrated to 
the serum powder of SMI with a vaccum concentrator (Savant SPD121P, Savant SpeedVac Concentrator, Thermo Scientific, USA) set at $4{ }^{\circ} \mathrm{C}$. Finally the serum powder of SMI was stored at $-80^{\circ} \mathrm{C}$ until analysis. Serum powder of SMI was dissolved in methanol and the solution was injected into HPLC system to analysis. Serum powder of SMI when dissolved in deionized water was used for the cells.

\section{Cells}

HUVECs were seeded at $5 \times 10^{4} \mathrm{~cm}^{-2}$ and cultured in DMEM supplemented with 100 units $\mathrm{mL}^{-1}$ penicillin, $100 \mu \mathrm{g} \mathrm{mL} \mathrm{m}^{-1}$ streptomycin, $20 \%(V / V)$ FCS and $10 \mathrm{ng} \mathrm{mL} \mathrm{m}^{-1} \mathrm{bFGF}$ for 24 hours. The medium was changed to DMEM supplemented with $0.1 \% \mathrm{BSA}, 10 \mathrm{ng} \mathrm{mL}^{-1}$ bFGF and various concentrations of cholestan- $3 \beta, 5 \alpha, 6 \beta$-triol. The number of cells in the confluent culture did not change significantly in the serum-free medium over the subsequent 48 hours unless triol was added. Cells between passages 2 to 7 were used for experiments.

HUVECs were stained with $500 \mathrm{ng} \mathrm{mL}^{-1}$ of DAPI for $10 \mathrm{~min}$ at room temperature. The number of apoptotic cells (approximately 1000-2000 cells per well) was counted for nine high power fields under a fluorescent microscope. Apoptotic cells were defined as cells with fragmented and/or more apoptotic bodies and their percentage was calculated.

\section{HPLC}

The HPLC equipment used was an Agilent 1100 series system (Agilent Technologies, USA) consisting of a binary pump, online vacuum degasser, autosampler, thermostated column compartment and multiple wavelength detection. A Tigerkin $\mathrm{C}_{18}$ column $(200 \times 4.6$ $\mathrm{mm}$ i.d., $5.0 \mu \mathrm{m}$ ) from Dalian Sipore Co. (China) was used for all chromatographic separations. The HPLC analytical conditions, which we developed before, were used in the assay. Linear gradient elution of eluents A [0.5 \% (V/V) aqueous formic acid] and B [0.5 \% (V/V) formic acid in acetonitrile] was used to run the separation. The first linear gradient was $5-20 \%$ eluent $B$ in the range of $0-10 \mathrm{~min}$, the second was $20-25 \%$ eluent $B$ in the range of $10-17 \mathrm{~min}$, and the last one was $25-55 \%$ eluent $B$ in the range of $17-35 \mathrm{~min}$. The system was restored to initial conditions after $5 \mathrm{~min}$. The solvent flow rate was $1.0 \mathrm{~mL} \mathrm{~min}^{-1}$, injection volume was $20 \mu \mathrm{L}$, and column temperature was maintained at $30{ }^{\circ} \mathrm{C}$. Chromatograms were recorded at $280 \mathrm{~nm}$ in the range of $0-13 \mathrm{~min}, 326 \mathrm{~nm}$ in the range of 13-23.5 $\mathrm{min}$, and at $286 \mathrm{~nm}$ in the range of $23.5-35 \mathrm{~min}$. Chemstation software (Agilent Technology) was used for peak detection and peak area calculation.

\section{Free radical scavenging activity and antioxidant activity of serum powder of SMI}

Free radical scavenging activity was determined as described by Blois (20). Briefly, acetate buffer (100 mmol L-1, pH 5.5), ethanol (100\%) and freshly prepared $500 \mathrm{nmol} \mathrm{L}^{-1}$ DPPH solution in ethanol were mixed in a volume ratio 1:1:0.5. After the test compound was added, the mixture was incubated at $25^{\circ} \mathrm{C}$ for $90 \mathrm{~min}$. The change in absorption at 517 nm was then determined with a spectrophotometer (TU-1901 double-beam UV-Vis spectrophotometer, China). 
Antioxidant properties were evaluated by measuring SOD activity. The experiment was performed using the SOD kit according to the manufacturer's instructions.

\section{Drug preparation}

SMI was prepared by the Qinchunbao Pharmaceutical Co., Ltd (China). It was made from the extract of Radix Salviae miltiorrhizae and prepared according to the standard injection production process. It was stored at $4{ }^{\circ} \mathrm{C}$. SMI was diluted with a $50 \%$ glucose injection in a volume ratio 1:5; the dilution should be freshly prepared prior to injecting it to the rats.

\section{Statistical analysis}

The data of free radical scavenging activity and antioxidant properties were presented as mean \pm standard error of the mean (SEM). Bivariate correlation was tested using the SPSS Version 17.0 software package (SPSS, USA) to analyze the correlation between the peak areas and antioxidant properties. Pearson correlation coefficients were obtained by bivariate correlation.

\section{RESULTS AND DISCUSSION}

\section{HPLC chromatograms of SMI serum powder}

Six groups of SMI serum powder were analyzed by HPLC. Tanshinol, protocatechuic aldehyde, rosmarinic acid, salvianolic acid B, protocatechuic acid and some metabolites were found in the serum powder of SMI (Fig. 1). As shown in Fig. 1 the retention time of peak $4 \mathrm{a}$ was close to the retention time of rosmarinic acid; however, this peak did not appear in the chromatogram of SMI. Thus it may be the metabolite of rosmarinic acid. Similar cases were peak $5 \mathrm{a}$ (the metabolite of salvianolic acid B) and peak $6 \mathrm{a}$ (the metabolite of salvianolic acid A). These metabolites and protocatechuic acid were found only in the serum powder of SMI. In addition, tanshinol, protocatechuic aldehyde, rosmarinic acid and salvianolic acid B which existed in SMI were also found in the serum powder of SMI (Table I). The ingredients that exist in the serum may be the active components. Therefore, tanshinol, protocatechuic aldehyde, rosmarinic acid, salvianolic acid B, protocatechuic acid and the metabolites may contribute to the efficacy of SMI.

\section{Serum powder of SMI inhibits endothelial cell apoptosis}

Serum powder of SMI prevented the triol-induced apoptosis of human umbilical vein endothelial cells in vitro (Fig. 2). In the assay, $5 \mu \mathrm{g} \mathrm{mL} \mathrm{m}^{-1}$ of triol induced apoptosis in HUVECs in a time-dependent manner in vitro. Triol induced significant apoptosis in HUVECs as early as $24 \mathrm{~h}$ from the start of incubation, and the number of apoptotic cells increased up to $48 \mathrm{~h}$. When $100 \mu \mathrm{g} \mathrm{mL}-1$ of SMI serum powder was added into the HUVECs with 5 $\mu \mathrm{g} \mathrm{mL} \mathrm{m}^{-1}$ of triol, the number of apoptotic cells decreased significantly (inhibition $47.6 \%$, $p<0.05$ ) vs. reference drug probucol (inhibition $72.1 \%, p<0.01$ ), in contrast to the blank control. In short, serum powder of SMI could inhibit the triol-induced apoptosis of HUVECs and protect normal HUVECs in vitro. 
a)

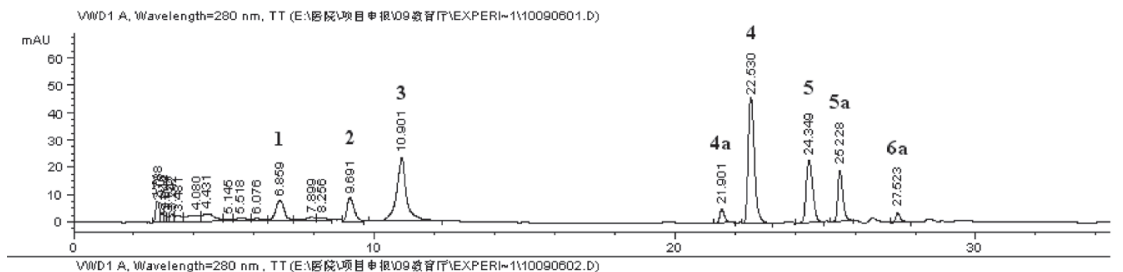

b)

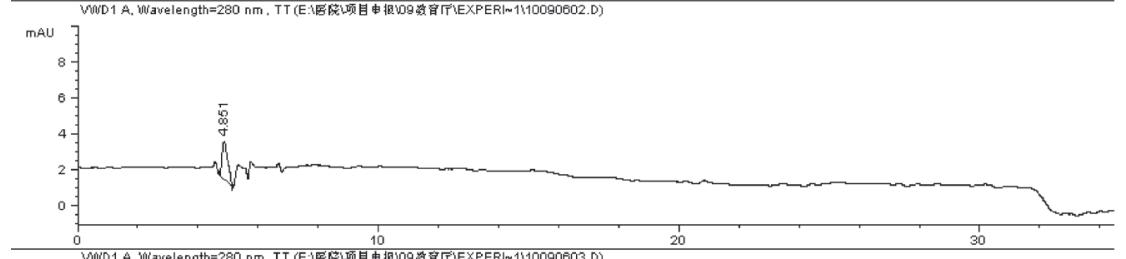

c)

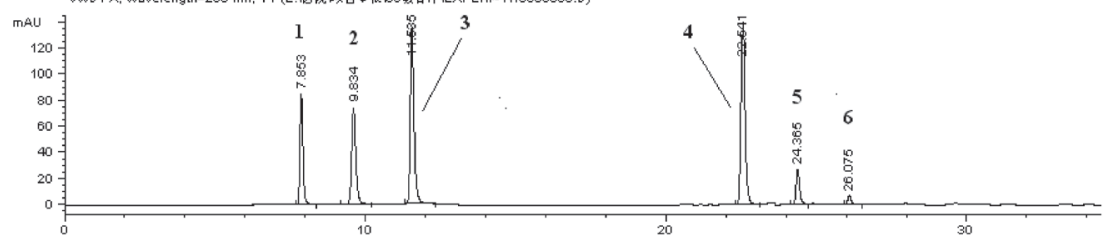

d)

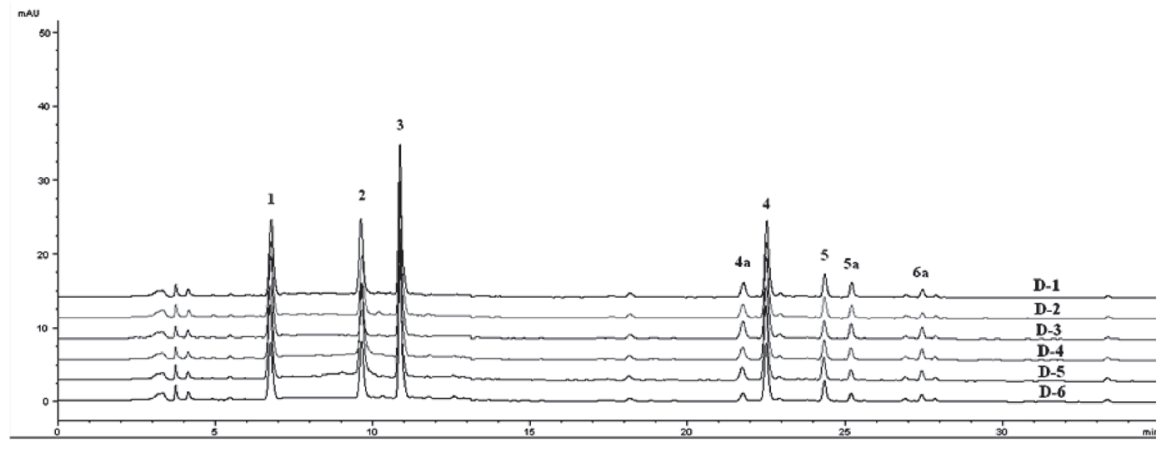

e)

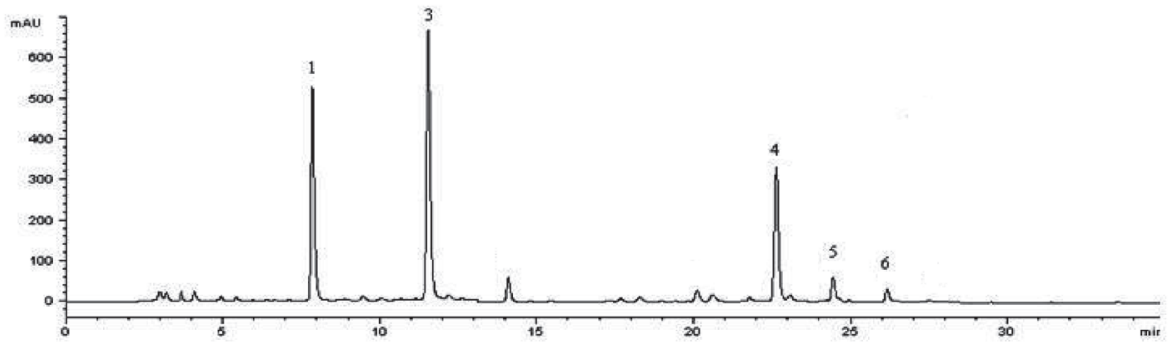

Fig. 1. HPLC chromatograms of: a) serum powder of SMI, b) blank control, c) standard, d) six groups of SMI serum powder (D-1 to D-6) and e) SMI. Components: 1 - tanshinol, 2 - protocatechuic acid, 3 protocatechuic aldehyde, 4 - rosmarinic acid, 5 - salvianolic acid B, 6- salvianolic acid A, 4a - metabolite of rosmarinic acid, 5a - metabolite of salvianolic acid B, $6 \mathrm{a}-$ metabolite of salvianolic acid A. 
Table I. Components in SMI and SMI serum powder

\begin{tabular}{lcc}
\hline Component & Content in SMI $\left(\mathrm{mol} \mathrm{L}^{-1}\right)^{\mathrm{a}}$ & Content in serum powder of SMI $\left(\mathrm{mol} \mathrm{L}^{-1}\right)^{\mathrm{a}}$ \\
\hline Tanshinol & $2.34 \pm 0.04$ & $(9.10 \pm 1.74) \times 10^{-2}$ \\
Protocatechuic aldehyde & $1.96 \pm 0.02$ & $(1.70 \pm 0.05) \times 10^{-2}$ \\
Salvianolic acid B & $0.42 \pm 0.01$ & $(1.56 \pm 0.04) \times 10^{-2}$ \\
\hline
\end{tabular}

${ }^{\mathrm{a}}$ Mean $\pm \operatorname{SD}(n=6)$.

Table II. Radical scavenging activity and SOD activity of SMI serum powder in HUVECs induced by triolb for 48 h by DPPH assay

\begin{tabular}{ccc}
\hline Group & $I C_{50}\left(\mu \mathrm{g} \mathrm{mL}^{-1}\right)$ & $\mathrm{SOD}\left(\mathrm{U} \mathrm{mL}^{-1}\right)$ \\
\hline 1 & $57.6 \pm 2.4^{c}$ & $0.53 \pm 0.05$ \\
2 & $52.4 \pm 1.9^{c}$ & $0.58 \pm 0.05^{c}$ \\
3 & $49.3 \pm 1.7^{c}$ & $0.63 \pm 0.03^{c}$ \\
4 & $54.7 \pm 2.2^{c}$ & $0.54 \pm 0.02$ \\
5 & $56.9 \pm 2.2^{c}$ & $0.51 \pm 0.05$ \\
6 & $58.3 \pm 2.9^{c}$ & $0.46 \pm 0.04$ \\
Negative control (triol) & $174.1 \pm 6.5^{c}$ & $0.21 \pm 0.04$ \\
Positive control (probucol) & $35.6 \pm 1.3^{c}$ & $0.79 \pm 0.03^{c}$ \\
\hline
\end{tabular}

Values were mean $\pm \operatorname{SEM}(n=6)$.

${ }^{\text {a }}$ Concentration of SMI: $100 \mu \mathrm{g} \mathrm{mL} \mathrm{m}^{-1}$.

${ }^{\mathrm{b}}$ Concentration of triol: $5 \mu \mathrm{g} \mathrm{mL} \mathrm{m}^{-1}$.

${ }^{\mathrm{c}} p<0.05$ compared with triol

Six groups of serum powder of SMI and probucol were added into the triol-induced apoptotic HUVECs. Apoptosis of endothelial cells was inhibited significantly $(p<0.05)$ in groups of SMI serum powder (Fig. 3).

Free radical scavenging activity and antioxidant activity of SMI serum powder

Serum powder of SMI exhibited free radical scavenging activity in the 1,1-diphenyl-2-picrylhydrazyl (DPPH) assay. The results are listed in Table II. As shown in Table II, all groups of SMI serum powder showed good radical scavenging activity, with the $I C_{50}$ values from $49.3 \pm 1.7$ to $58.3 \pm 2.9 \mu \mathrm{g} \mathrm{mL}^{-1}$ in contrast to triol $\left(174.1 \pm 6.5 \mu \mathrm{g} \mathrm{mL}{ }^{-1}\right)$. Groups 3 and 2 were the best. The radical scavenging activity of serum powder of SMI was 1.5 times lower than that of the reference drug probucol $\left(35.6 \pm 1.3 \mu \mathrm{g} \mathrm{mL} \mathrm{mL}^{-1}\right)$. 


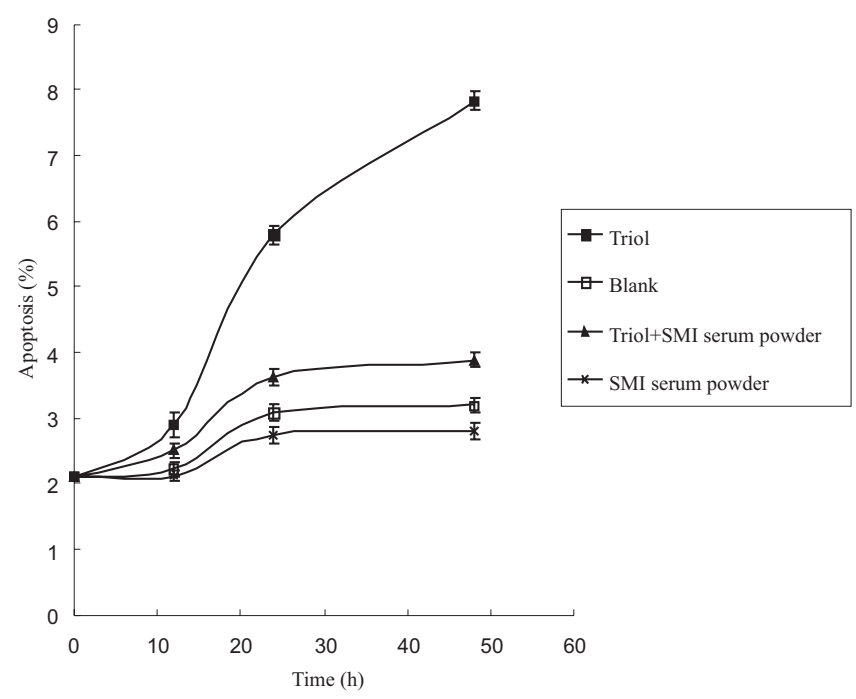

Fig. 2. The time course for HUVECs cultured in SMI serum powder (X-type), SMI serum powder with

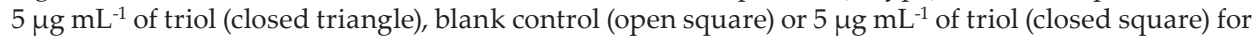
$48 \mathrm{~h}$. Each point represents the mean $\pm \operatorname{SEM}(n=6)$.

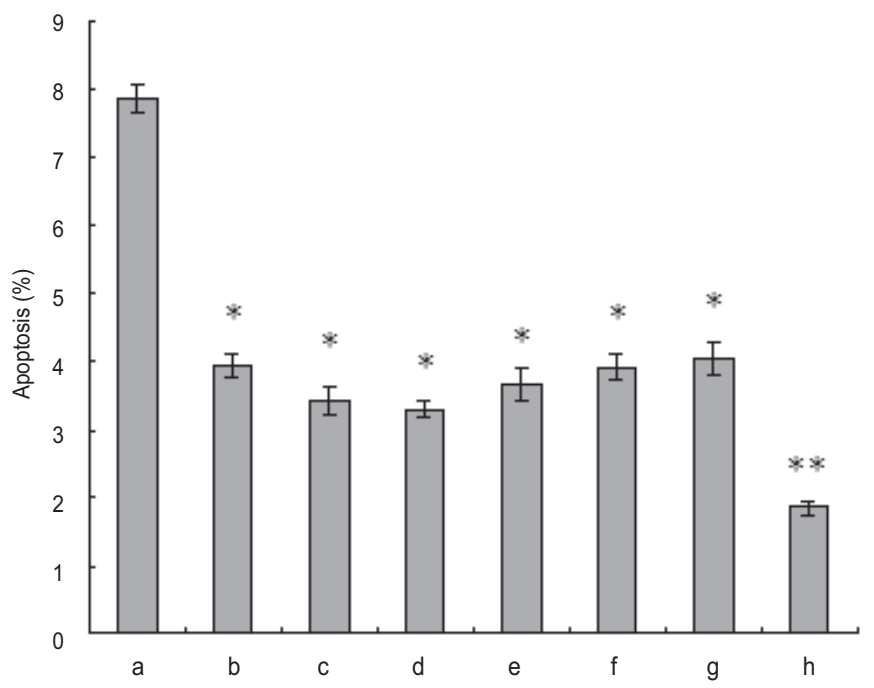

Fig. 3. The effect of SMI serum powder on the triol-induced apoptosis of HUVECs. a - triol, b-group D-1 (5 min), c - group D-2 (10 min), d - group D-3 (15 min), e - group D-4 (20 min), f - group D-5 $(25 \mathrm{~min}), \mathrm{g}$ - group D-6 (30 min), h - probucol. Each point represents the mean $\pm \mathrm{SE}(n=6)$. Significantly different from triol: ${ }^{*} p<0.05,{ }^{* *} p<0.01$. 
J. Shen et al:: Analysis of active components in Salvia miltiorrhiza injection based on vascular endothelial cell protection, Acta Pharm. 64 (2014) 325-334.

Table III. Pearson correlation coefficient between peak area and antioxidant property of ingredients in SMI serum powder

\begin{tabular}{lccc}
\hline Compound & $\begin{array}{c}\text { Retention time } \\
(\mathrm{min})\end{array}$ & $\begin{array}{c}\text { Peak area vs. } \\
I C_{50}\end{array}$ & $\begin{array}{c}\text { Peak areas vs. } \\
\text { SOD }\end{array}$ \\
\hline Tanshinol & 7.899 & $-0.880^{\mathrm{a}}$ & $0.861^{\mathrm{a}}$ \\
Protocatechuic acid & 9.691 & 0.398 & -0.440 \\
Protocatechuic aldehyde & 10.901 & $-0.722^{\mathrm{a}}$ & $0.807^{\mathrm{a}}$ \\
Metabolite of rosmarinic acid & 21.901 & 0.005 & -0.139 \\
Rosmarinic acid & 22.530 & -0.773 & 0.654 \\
Metabolite of salvianolic acid B & 23.388 & -0.227 & 0.129 \\
Salvianolic acid B & 24.349 & $-0.954^{\mathrm{b}}$ & $0.911^{\mathrm{a}}$ \\
Metabolite of salvianolic acid A & 27.523 & -0.449 & 0.279 \\
\hline
\end{tabular}

a Significance level of correlation $(p<0.05)$

b Significance level of correlation $(p<0.01)$.

The activity of superoxide dismutase was used as an index of oxidative damage in the assay. All groups of serum powder of SMI increased the low activity of SOD caused by triol (SOD value of $\left.0.21 \pm 0.04 \mathrm{U} \mathrm{mL}^{-1}\right)$. As shown in Table II, group 3 was better $(0.63 \pm 0.03$ $\mathrm{U} \mathrm{mL}^{-1}$ ) than the other groups.

Correlation analysis based on the chromatograms and antioxidant properties of SMI serum powder

According to the results shown in Table III, the area of four peaks in HPLC chromatograms showed higher correlation to radical scavenging activity than the others, with the absolute correlation coefficient value higher than 0.7. These four compounds were salvianolic acid B, tanshinol, protocatechuic aldehyde and rosmarinic acid. Among them, salvianolic acid $B$ reached the significance level with $p<0.01$ and tanshinol and protocatechuic aldehyde with $p<0.05$. Since correlation between $I C_{50}$ and peak area appeared in protocatechuic acid and rosmarinic acid metabolite, we suggest that the two components had no free radical scavenging activity. Neither did metabolites of salvianolic acid $\mathrm{B}$ and $\mathrm{A}$, due to their low $R$. Peak areas of salvianolic acid B, tanshinol, protocatechuic aldehyde and rosmarinic acid showed higher correlation to SOD activity than the other components (Pearson correlation coefficient $R>0.6$ ). Among them, salvianolic acid $\mathrm{B}$, tanshinol and protocatechuic aldehyde reached the significance level with $p<0.05$. Our findings suggest that protocatechuic acid and the rosmarinic acid metabolite had almost no antioxidant activity. Furthermore, low $R$ between peak area and SOD activity suggested that metabolites of salvianolic acid B and A were unrelated to antioxidant activity.

This method enables rapid identification of active components in herbal medicine and provides a meaningful tool for their quality control. 


\section{CONCLUSIONS}

Based on these results, it was concluded that salvianolic acid B, tanshinol and protocatechuic aldehyde correlated well with the antioxidant properties of serum powder of SMI. They contributed to the protection of vascular endothelial cells by inhibiting the triol-induced apoptosis of HUVECs and protecting normal HUVECs in vitro. They were shown for the first time to be antioxidants by correlation analysis. Salvianolic acid B was demonstrated to be the most potential antioxidant of all the SMI ingredients, since it was closely related to the free radical scavenging activity and SOD activity with the Pearson correlation coefficient higher than 0.9. In future experiments, we will identify the metabolites of SMI and a systematic study of pharmacokinetics of SMI will be carried out.

Acknowledgements. - This research was financially supported by the National TCM Expert Heritage Studio Project, China (No. State Administration of TCM [2013] 47), Chinese Medical Science and Technology Projects of Zhejiang Province (No. 2010ZJ001), TCM Expert Heritage Studio Project of Zhejiang Province (No. Health and Family Planning Commission of Zhejiang Province [2012] 210), Clinical Pharmacy Research Fund of Zhejiang Province Association of Chinese Integrative Medicine, (No. 2013LYSX018), Scientific Research Fund of Zhejiang Provincial Education Department (No. Y200906846) and the program for Chuying Talent of the First Affiliated Hospital of Zhejiang Chinese Medical University (No. RS02/2012).

\section{REFERENCES}

1. S. Bent and R. Ko, Commonly used herbal medicines in the United States: a review, Am. J. Med. 116 (2004) 478-485; DOI: 10.1016/j.amjmed.2003.10.036.

2. T. O. Cheng, Danshen: what every cardiologist should know about this Chinese herbal drug, Int. J. Cardiol. 110 (2006) 411-412; DOI: 10.1016/j.ijcard.2005.08.069.

3. L. Zhou, Z. Zuo and M. S. Chow, Danshen: An overview of its chemistry, pharmacology, pharmacokinetics, and clinical use, J. Clin. Pharmacol. 45 (2005) 1345-1359; DOI: 10.1177/0091270005282630.

4. Y. X. Chang, D. M. Yan, L. L. Chen, X. P. Ding, J. Qi, L. Y. Kang, B. L. Zhang and B. Y. Yu, Potency fingerprint of herbal products Danshen Injection for their quality evaluation, Chem. Pharm. Bull. 57 (2009) 586-590; DOI: 10.1248/cpb.57.586.

5. J. H. Ho and C. Y. Hong, Salvianolic acids: small compounds with multiple mechanisms for cardiovascular protection, J. Biomed. Sci. 18 (2011) 30-35; DOI: 10.1186/1423-0127-18-30.

6. Z. Xia, J. Gu, D. M. Ansley, F. Xia and J. Yu, Antioxidant therapy with Salvia miltiorrhiza decreases plasma endothelin-1 and thromboxane B2 after cardiopulmonary bypass in patients with congenital heart disease, J. Thorac. Cardiovasc. Surg. 126 (2003) 1404-1410; DOI: 10.1016/S00225223(03)00970-X.

7. Y. Z. Zhu, S. H. Huang, B. K. Tan, J. Sun, M. Whiteman and Y. C. Zhu, Antioxidants in Chinese herbal medicines: a biochemical perspective, Nat. Prod. Rep. 21 (2004) 478-489; DOI: 10.1039/ B304821G.

8. J. L. Zhang, M. Cui, Y. He, H. L. Yu and D. A. Guo, Chemical fingerprint and metabolic fingerprint analysis of Danshen injection by HPLC-UV and HPLC-MS methods, J. Pharm. Biomed. Anal. 36 (2005) 1029-1035; DOI: 10.1016/j.jpba.2004.09.009.

9. F. Qiu, R. Zhang, J. Sun, A. Jiye, H. Hao, Y. Peng, H. Ai and G. Wang, Inhibitory effects of seven components of Danshen extract on catalytic activity of cytochrome P450 enzyme in human liver microsomes, Drug Metab. Dispos. 36 (2008) 1308-1314; DOI: 10.1124/dmd.108.021030. 
10. D. G. Kang, Y. G. Yun, J. H. Ryoo and H. S. Lee, Anti-hypertensive effect of water extract of Danshen on renovascular hypertension through inhibition of the renin angiotensin system, Am. J. Chin. Med. 30 (2002) 87-93; DOI: 10.1142/S0192415X02000107.

11. G. R. Zhao, H. M. Zhang, T. X. Ye, Z. J. Xiang, Y. J. Yuan, Z. X. Guo and L. B. Zhao, Characterization of the radical scavenging and antioxidant activities of Tanshinol and salvianolic acid B, Food Chem. Toxicol. 46 (2007) 73-81; DOI: 10.1016/j.fct.2007.06.034.

12. Y. Sun, H. Zhu, J. Wang, Z. Liu and J. Bi, Isolation and purification of salvianolic acid A and salvianolic acid B from Salvia miltiorrhiza by high-speed counter-current chromatography and comparison of their antioxidant activity, J. Chromatogr. B 877 (2009) 733-737; DOI: 10.1016/j. jchromb.2009.02.013.

13. J. Z. Xu, J. Shen, Y. Y. Cheng and H. B. Qu, Simultaneous detection of seven phenolic acids in Danshen injection using HPLC with ultraviolet detector, J. Zhejiang Univ. Sci. B 9 (2008) 728-733; DOI: 10.1631/jzus.B0820095.

14. Y. X. Chang, X. P. Ding, J. Qi, J. Cao, L. Y. Kang, D. N. Zhu, B. L. Zhang and B. Y. Yu, The antioxidant-activity-integrated fingerprint: an advantageous tool for the evaluation of quality of herbal medicines, J. Chromatogr. A 1208 (2008) 76-82; DOI: 10.1016/j.chroma.2008.08.054.

15. D. Zhao, D. E. Han, N. Li, Y. Lu, T. T. Li, S. Y. Yang, J. K. He and X. J. Chen, Simultaneous determination of six phenolic constituents of Danshen injection in rat plasma by LC-ESI-MS and its application to a pharmacokinetic study, Eur. J. Mass Spectrom. (Chichester) 17 (2011) 395-403; DOI: 10.1255/ejms.1137.

16. R. Xia and X. Chen, Effects of Danshen injection on the malignant obstructive jaundice in the SD rat model, J. Huazhong Univ. Sci. Technol. Med. Sci. 26 (2006) 686-689; DOI: 10.1007/s11596-006-06153.

17. Z. You, Y. Xin, Y. Liu, B. Han, L. Zhang, Yi. Chen, Yu. Chen, L. Gu, H. Gao and Y. Xuan, Protective effect of Salvia Miltiorrhizae injection on N(G)-nitro-D-arginine induced nitric oxide deficient and oxidative damage in rat kidney, Exp. Toxicol. Pathol. 64 (2012) 453-458; DOI: 10.1016/j.etp.2010.10.013.

18. D. Y. Gao, L. M. Han, L. H. Zhang, X. L. Fang and J. X. Wang, Bioavailability of salvianolic acid B and effect on blood viscosities after oral administration of salvianolic acids in beagle dogs, Arch. Pharm. Res. 32 (2009) 773-779; DOI: 10.1007/s12272-009-1517-2.

19. L. Shen, L. Zhang, Y. Feng, D. S. Xu and X. Lin, Study on fingerprints correlated with pharmacodynamic of Paeonia lacliflora and Glycyrrhiza uralensis effective compounds, Chin. J. Chin. Mater. Med. 33 (2008) 2658-2662; DOI: 10.4268/cjcmm 200822021.

20. M. S. Blois, Antioxidant determination by the use of a stable free radical, Nature 181 (1958) 1199_ 1200 . 